\title{
Investigating host-bacterial interactions among enteric pathogens
}

Tungadri Bose ${ }^{1,2}$, K. V. Venkatesh ${ }^{2}$ and Sharmila S. Mande ${ }^{1^{*}}$

\begin{abstract}
Background: In 2017, World Health Organization (WHO) published a catalogue of 12 families of antibiotic-resistant "priority pathogens" that are posing the greatest threats to human health. Six of these dreaded pathogens are known to infect the human gastrointestinal system. In addition to causing gastrointestinal and systemic infections, these pathogens can also affect the composition of other microbes constituting the healthy gut microbiome. Such aberrations in gut microbiome can significantly affect human physiology and immunity. Identifying the virulence mechanisms of these enteric pathogens are likely to help in developing newer therapeutic strategies to counter them.

Results: Using our previously published in silico approach, we have evaluated (and compared) Host-Pathogen Protein-Protein Interaction (HPI) profiles of four groups of enteric pathogens, namely, different species of Escherichia, Shigella, Salmonella and Vibrio. Results indicate that in spite of genus/ species specific variations, most enteric pathogens possess a common repertoire of HPIs. This core set of HPIs are probably responsible for the survival of these pathogen in the harsh nutrient-limiting environment within the gut. Certain genus/ species specific HPIs were also observed.

Conslusions: The identified bacterial proteins involved in the core set of HPIs are expected to be helpful in understanding the pathogenesis of these dreaded gut pathogens in greater detail. Possible role of genus/ species specific variations in the HPI profiles in the virulence of these pathogens are also discussed. The obtained results are likely to provide an opportunity for development of novel therapeutic strategies against the most dreaded gut pathogens.
\end{abstract}

Keywords: Gut pathogens, Host pathogen interactions, Protein interaction networks, Therapeutic targets

\section{Background}

The human gut harbors more than 100 trillion microbial cells belonging to over a 1000 phylotypes $[1,2]$. This microbial community, referred to as the 'human gut microbiota', is known to impact human physiology, metabolism, nutrition and immune functions [1,3]. The human gut (enteric) microbiota is predominated by commensal species belonging to phyla Firmicutes and Bacteroidetes [1,3]. Although pathogenic bacteria (like Campylobacter jejuni, Salmonella enterica, Vibrio cholera, Escherichia coli, and Bacteroides fragilis) are also observed in gut microbiota, their abundances are significantly lower in healthy human gut [3]. Interestingly, species like Helicobacter pylori, which

\footnotetext{
* Correspondence: sharmila.mande@tcs.com

${ }^{1}$ Bio-Sciences R\&D Division, TCS Innovation Labs, Tata Consultancy Services

Limited, Pune, India

Full list of author information is available at the end of the article
}

are otherwise commensal (and are constituents of the healthy gut microbial community), can acquire pathogenic phenotype under certain conditions [3]. The manifestation of virulence in pathogenic bacteria is usually mediated through small molecules (such as bacterial toxins) and cell surface associated and secreted proteins, which in turn interact with the host proteins.

In 2017, World Health Organization (WHO) published a list of pathogens [4] perceived to pose greatest threat to humanity. The report identified growing antimicrobial resistance of these pathogens as the major cause of concern. The associations between the host and various pathogens are governed by several factors which include expression patterns of bacterial gene/ protein as well as the availability of metabolites in the environmental niche that is inhabited by the pathogens. Studying 'HostPathogen Protein-Protein Interactions' (HPIs) can help 
in understanding (at least in part) the probable mechanisms of infections adopted by different pathogens $[5,6]$. Notably, six of the 12 WHO enlisted pathogens [4] correspond to enteric diseases, thereby highlighting the need to understand the probable biological mechanisms of the enteric pathogens (including their interactions with host) in greater detail.

HPI studies may either focus on an in-depth understanding of a particular aspect of pathogenesis or a host defense mechanism involving one (or a few) protein(s) from the host and pathogen [7-10], or may aim at obtaining a systems level view of the host-pathogen interplay [11-14]. While the former relies primarily on experimental approaches to validate a hypothesis, the latter can be studied using genome-scale bioinformatic approaches. The said bioinformatics approaches typically rely on a template protein-protein interaction (PPI) library for inferring HPIs among the host and a pathogen $[5,6,14]$. HPIs from template PPI libraries can either be inferred using (a) structural similarity [15-19] and/ or by (b) sequence similarity based methods $[5,6,14,20$, 21]. Thus the choice of tool to study HPI is usually determined by the question(s) to be addressed. The objective of the current study was to perform an in silico comparative analysis of the HPI profile of different gut pathogens. The current investigation therefore used a genome-scale bioinformatics approach. Given the scarcity (and un-uniformity) in the availability of good quality 3-D structures of human proteins as well as of all the studied pathogens, a sequence similarity based methodology was preferred over a structural similarity based approach.

In our earlier published study, we reported that different strains of $E$. coli share a common repertoire of interactions with the host, irrespective of the pathogenic nature [5]. Parasites with similar sites of infection have also been shown to share common features in their host-parasite interaction networks [22]. Since all microbes residing in the human gut share a common environment, we speculated that such similarities in HPI profiles could also exist among most (if not all) bacterial groups residing in human gut. The HPI profile of different enteric pathogens were accessed to check if there were any underlying commonalities in their interactions with the host. Such findings could be utilized for devising broad-spectrum antimicrobial strategies against these pathogens. In this work, we have performed an in silico analysis of HPIs pertaining to different enteric pathogens (namely, different species of Escherichia, Shigella, Salmonella and Vibrio) with an objective to decode their pathogenic mechanisms. We could identify a common repertoire of bacterial protein which is possibly involved in the microbes' adaptation to the niche environment within the gut. In addition, bacterial proteins involved in genus/ strain specific HPIs with the host could also be found. This identified set of bacterial proteins may be used as potential therapeutic targets for narrowspectrum antimicrobial development. Thus knowledge obtained from this study is likely to help in better understanding of the virulence processes adopted by different enteric pathogens and is likely to provide a road map for future studies in this direction.

\section{Results \\ Host-pathogen interactions (HPIs) involving human cells and enteric pathogens}

The number of host-pathogen interactions identified for each of the studied strains is presented in Table 1 (details in Additional file 1). The number of host and bacterial proteins involved in the HPIs was observed to be substantially lower in $V$. cholerae strains as compared to other pathogens. Further, in concordance with their generic phenotypes [23], the number of interactions pertaining to Shigella strains was noted to be similar to those of $E$. coli. However, interaction patterns involving $S$. enterica strains were found to differ from that in E. coli. Notably, as compared to the S. enterica serovar Typhi species the S. enteric serovar Typhimurium species demonstrated a higher number of HPIs with the human. The possible roles of this additional set of HPIs in S. enteric serovar Typhimurium infection has been discussed in a later section. In line with an earlier literature [5], a number of host and pathogen proteins were seen to have high degrees of interaction in the corresponding host-pathogen interaction networks (Additional file 2). As was indicated in our earlier study [5], such high degree nodes are usually associated with crucial biological functions. Interestingly, some of these high degree nodes (in the present study) were found to be absent in some of the analyzed HPI networks. For example, amongst the studied enteric pathogens, HPIs involving UDP-sugar hydrolase (UshA) in S. enterica serovar Typhi strains was absent. Similarly, HPIs involving acyl-CoA thioesterase I (TesA) was absent in Shigella flexneri 301. Also the HPI sub-network involving three high degree bacterial proteins (AbcT2, AbcT3, AbcT5) belonging to the HlyB subfamily of $A B C$ transporter in two studied $V$. cholerae strains (O395 and N16961) were found to be absent in the HPI profiles of all the other studied bacterial strains. It is to be noted that HlyB family of $A B C$ transporter proteins have previously been shown to play vital roles in the secretion of hemolysins which is crucial for invasion of the host's intestinal villi by $V$. cholerae [24].

In general, the host and the bacterial proteins which demonstrated high degree centrality in the HPI-network were also characterized by high betweenness centralities. However, certain bacterial and human proteins were found to have high betweenness values in spite of their low degree centralities (Additional file 2). The 
Table 1 Statistics of the number of observed protein-protein interactions (PPIs) involving human (host) and bacterial (pathogen) proteins, among the studied bacterial strains

\begin{tabular}{|c|c|c|c|c|c|}
\hline Interacting bacterial strain & $\begin{array}{l}\text { Total number of } \\
\text { interactions }\end{array}$ & $\begin{array}{l}\text { Total number of } \\
\text { proteins involved }\end{array}$ & $\begin{array}{l}\text { Number of interacting } \\
\text { bacterial proteins }\end{array}$ & $\begin{array}{l}\text { Number of interacting } \\
\text { human proteins }\end{array}$ & $\begin{array}{l}\text { Average degree of } \\
\text { nodes (proteins) }\end{array}$ \\
\hline $\begin{array}{l}\text { Salmonella enterica subsp. } \\
\text { enterica serovar Typhi CT18 }\end{array}$ & 582 & 273 & 104 & 169 & 4.20 \\
\hline $\begin{array}{l}\text { Salmonella enterica subsp. } \\
\text { enterica serovar Typhi Ty2 }\end{array}$ & 582 & 273 & 104 & 169 & 4.20 \\
\hline $\begin{array}{l}\text { Salmonella enterica Serovar } \\
\text { Typhimurium LT2 }\end{array}$ & 634 & 279 & 109 & 170 & 4.48 \\
\hline Escherichia coli O157 H7 EC4115 & 762 & 283 & 118 & 165 & 5.29 \\
\hline Escherichia coli O157 H7 EDL933 & 773 & 292 & 122 & 170 & 5.21 \\
\hline Escherichia coli O157 H7 Sakai & 771 & 289 & 120 & 169 & 5.25 \\
\hline $\begin{array}{l}\text { Escherichia coli O157 H7 } \\
\text { TW14359 }\end{array}$ & 764 & 286 & 120 & 166 & 5.25 \\
\hline Escherichia coli K-12 MG1655 & 783 & 291 & 120 & 171 & 5.29 \\
\hline Shigella dysenteriae & 744 & 265 & 102 & 163 & 5.52 \\
\hline $\begin{array}{l}\text { Shigella flexneri } 301 \text { (serotype } \\
\text { 2a) }\end{array}$ & 703 & 269 & 108 & 161 & 5.14 \\
\hline $\begin{array}{l}\text { Vibrio cholerae O1 biovar El Tor } \\
\text { N16961 }\end{array}$ & 346 & 154 & 54 & 100 & 4.49 \\
\hline Vibrio cholerae O395 & 357 & 155 & 55 & 100 & 4.61 \\
\hline
\end{tabular}

topological architecture of such proteins (nodes) in the HPI-network was indicative of their central role in the infection process [5]. Noticeably, betweenness centralities values of some of the human proteins, such as $\mathrm{O}$ sialoglycoprotein endopeptidase like 1 (OSGEPL1), Vtype proton ATPase subunit B (ATP6V1B2) and acid phosphatase 1 (ACP1) were seen to vary between the studied strains (biological implications are discussed later). The above observations are probably indicative of the selective adaptation of different groups of bacterial pathogens to survive and persist inside the host.

In an attempt to understand the probable role of the identified HPIs in the overall infection process for each of the studied organisms, analyses of the KEGG infection pathways with respect to the human proteins involved in the HPIs were performed. The KEGG database [25, 26] contains literature curated information on the human proteins that are affected during bacterial infection processes. Since information pertaining to $V$. cholerae infection process was not available in the database, the analyses were restricted to understanding the virulence processes of E. coli, S. enterica and Shigella. It was observed that for all the studied organisms (Additional file 3: Appendix 1), there was a significant overlap between the human protein set which interacted with the pathogens (as per the HPI analysis) and the human proteins which were involved in the infection process (reported in KEGG). A few genus and species specific differences were also observed. For example, proteins associated to human Toll-like receptor signaling pathway was found to be more intricately associated with the E. coli infection process as compared to infection by other pathogens. Further, mechanism of actin rearrangement of human epithelial cells was found to be different in response to $S$. flexneri infection when compared with $S$. dysenteriae infection.

\section{Common interactions between host and different enteric pathogens}

Irrespective of their mode of infection, all enteric pathogens enter the host alimentary system and adapt to the harsh environment inside the human gut. Upon entry inside the host, these pathogens need to cope up with the host's bile acids, immune defenses as well as a nutrient scarce environment. It was therefore speculated that the enteric pathogens probably adopt similar strategies (including their interactions with host) to deal with the adversities posed by their host. Analysis of the HPI profiles of the studied pathogens revealed a total of 122 PPIs which were common to most of the studied pathogens (Fig. 1). The interactions included bacterial proteins like UDP-sugar hydrolase (UshA), arginine-binding periplasmic protein 1 (ArtI), transferrin binding protein A (TbpA), cytosol aminopeptidase (PepA), cytosol non-specific dipeptidase (PepD), aminopeptidase $\mathrm{N}(\mathrm{PepN})$, glutathione reductase (Gor), glutathione synthetase (GshB), ABC transporter periplasmic-binding protein (SapA), gammaglutamyltransferase (Ggt) etc. (Additional file 4A). Experimental evidences supporting the functional importance of a few of these bacterial proteins and/ or their involved in interactions with the host could be obtained from earlier 


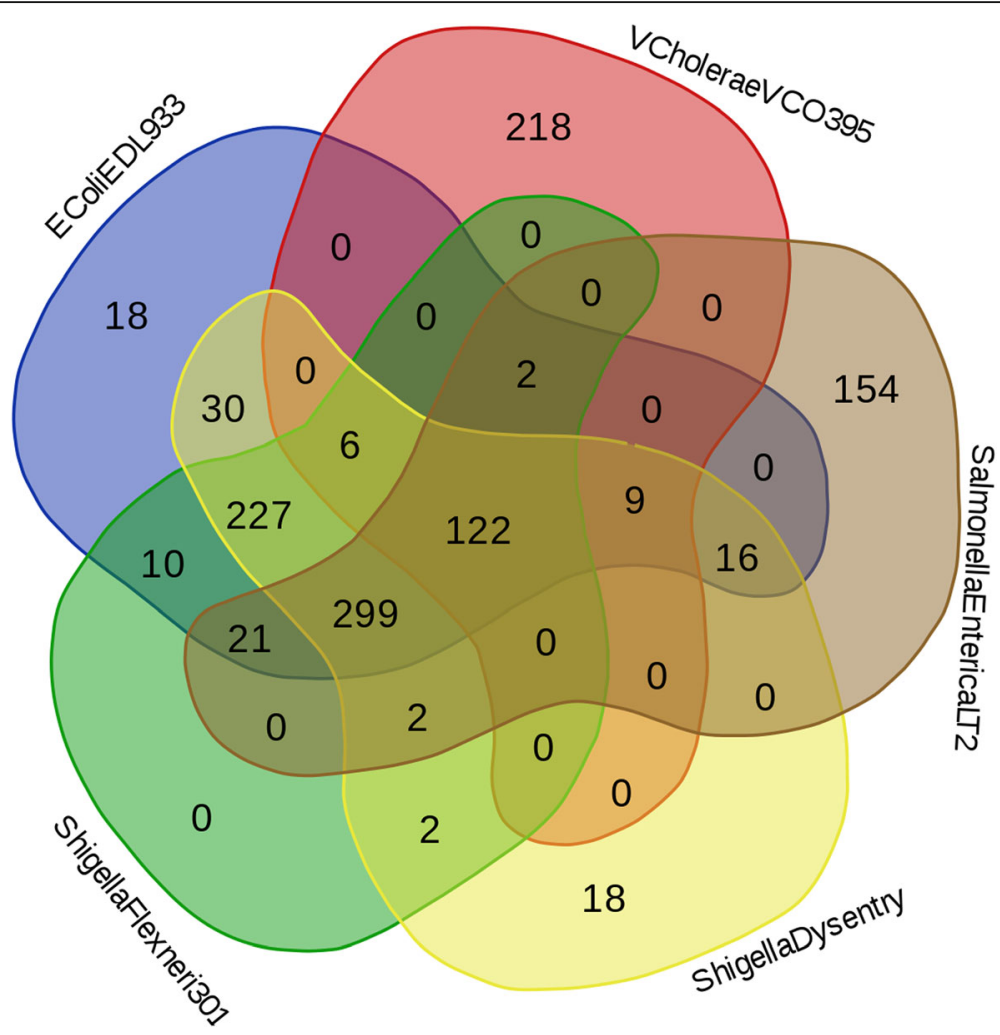

Fig. 1 Euler diagram representing the number of HPIs involving human proteins and those from the different studied enteric pathogens. A total of 122 PPIs involving 17 bacterial and 122 human proteins were common to all the studied pathogens

literatures. For example, significant reduction in CFUs of $S$. enterica serovar Typhimurium in aminopeptidase $\mathrm{N}(\mathrm{PepN})$ mutants as compared to wild type was reported in a study which inspected systemic infection in mice models [27]. Further, the bacterial proteins involved in this subset of interactions were found to be enriched in glutathione and sulfur metabolism (Additional file 4A). Given the role of glutathione in innate immunity and inflammation [28], the ability to abrogate glutathione mediated stress is probably important for a pathogen to survive inside the host. Further, sulfur containing compounds are often found to be associated with biological pathways leading to detoxification of reactive oxygen species (ROS) and glutathione [29, 30]. Moreover, in line with the expectations, bacterial superoxide dismutase (SodB) was also found to occur among the core interactors in the HPI networks. The other bacterial proteins in this sub-network were UshA and SapA. While UshA is involved in abrogation of host immune defenses [31-33], SapA has been shown to be play roles in neutralization of antimicrobial peptides (AMPs) [34].

The human proteins involved in this subset of interactions were found to be enriched in ATP driven transmembrane movement of substances, nucleotide metabolism and cofactor metabolism (Additional file 4B). The average degree centrality of these human proteins was observed to be substantially lower than the rest of the nodes (proteins) in the network. In order to evaluate whether this set of interactions was biologically meaningful or simply an artifact, this subset of proteins (along with their 1st degree neighbors) was plotted on the KEGG infection pathway. The proteins were found to be associated with the mechanism related to activation of inflamsomes in intestinal epithelium cells. This was also elucidated in a few previously published literatures [35-38]. The probable role of these human proteins (which were observed in the core set of HPIs with enteric pathogens and their neighbors) in the mechanism of activation of inflamsomes in intestinal epithelium cells is illustrated in Fig. 2. While the host proteins involved in the HPIs is likely to play key roles in the initiation of the immune responses, the bacterial proteins participating in these HPIs could potentially aid the pathogen in evading host immunity in order to successfully colonize inside the host.

\section{Variations in interactions (with host) of enteric pathogens belonging to the same genera}

The HPI profiles of the studied pathogens consisted of a core set of 122 interactions between the host and pathogen proteins (Fig. 1). Several genera and species-specific interactions were also observed (Figs. 3, 4, 5 and Additional file 3: Figure S1). Some of the observed differences (as compared to $E$. coli) are presented below. 


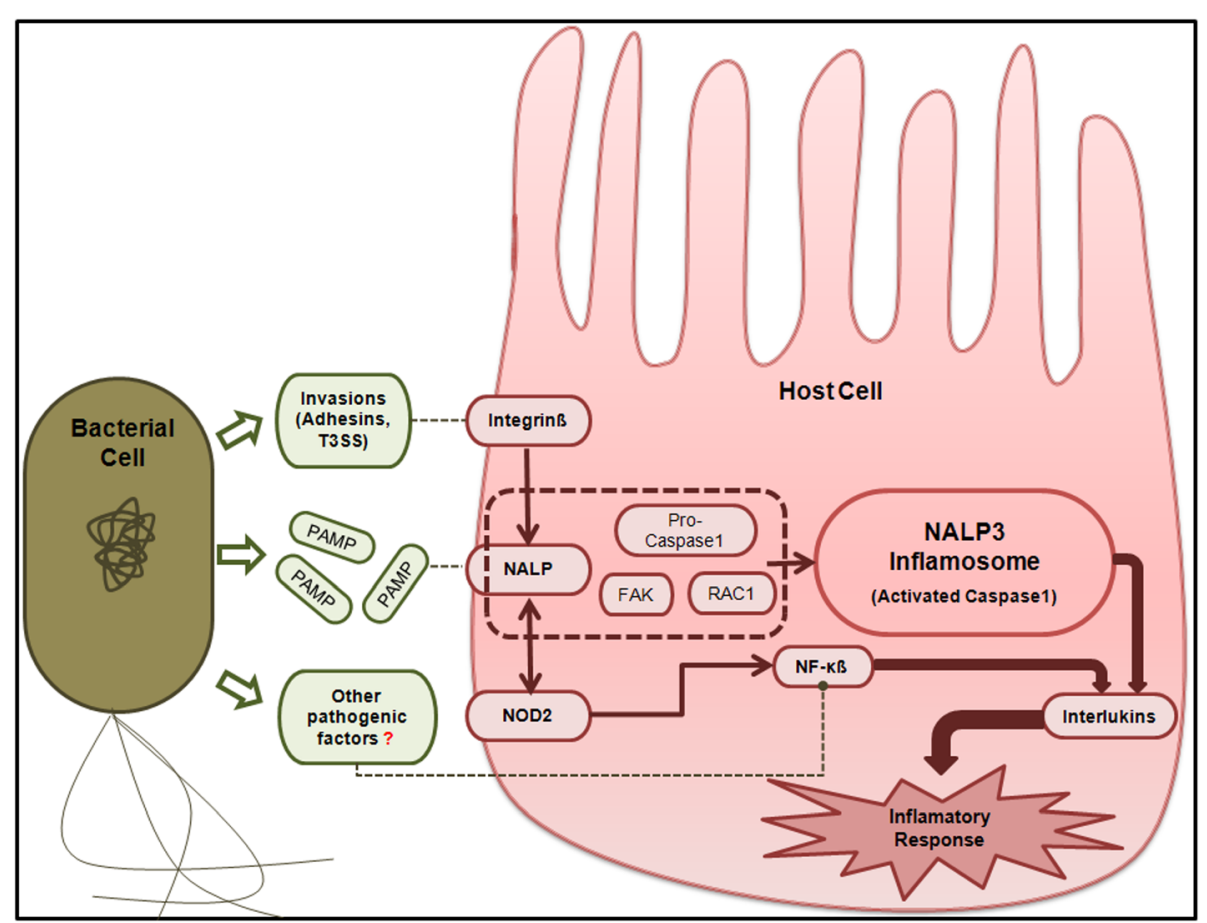

Fig. 2 Probable role of the human proteins (and their neighbors), involved in the core set of HPIs with enteric pathogens, in the activation of inflamsomes in intestinal epithelium cells. NALP3 is a pathogen recognition receptor of the NOD-like receptor (NLR) subfamily. It functions by recognizing pathogen-associated molecular patterns (PAMPS). NALP3 together with proteins like PYCARD/ ASC forms a caspase-1 activation complex known as the NALP3 inflamasome. Activation of NALP3 inflamasome further requires the assistance of focal adhesion kinase and rac 1 from the focal adhesion complex signaling pathway. Interaction of host integrin $\beta$ with pathogenic factors (like invasions) acts as the first signal for the activation of NALP3 inflamasomes. The second set of signals for the activation of inflamosomes is mediated through the Type III secretion system translocon. Expression of NALP3 inflamasome in turn results in the release of interleukins, especially IL-18. NALP3 also interacts with NOD2 leading to activation of interleukins through a pathway independent of the caspase recruitment domain-containing proteins. Inhibition of NF-KB is a common strategy adopted by enteric pathogens to block the integrin signaling pathway, thereby evading host's immune and inflammatory responses.

\section{Interactions of Shigella (with host) that are unique with respect to interactions of E. coli strains (with host)}

The HPI profiles pertaining to both $S$. dysenteriae and $S$. flexneri comprised of interactions involving a putative iron transport protein SitA (Fig. 3 and Additional file 3: Figure S2). The putative iron transport gene SitA in S. flexneri (as well as several commensal enteric bacterial groups) is known to get induced during the intracellular survival stage $[39,40]$. Notably, SitA was found to interact with human ATP-dependent mitochondrial porphyrin importer protein (ABCB6), also known for its role in iron metabolic pathways [41]. Given that the in vivo survival of Shigella is dependent on acquisition of essential nutrients (such as iron) from the host, the observed interaction between the bacterial putative iron transport protein (SitA) and host ATP-dependent mitochondrial porphyrin importer protein $(\mathrm{ABCB} 6)$ appears to be important. It may be noted that although SitA was found to be present in the HPI profile of the virulent Shigella strains, it is also known to be abundance among non-pathogenic (enteric) bacterial groups $[39,40]$. Therefore, this putative iron transport protein probably aids the bacteria in nutrient (iron) acquisition from the host and may not be considered as a 'pathogenic' factor $[39,40]$. The studied Shigella strains were also seen to be involved in 17 additional interactions (Fig. 3 and Additional file 3: Figure S1). Interestingly, while similar interactions were also observed in the pathogenic strain of $E$. coli (EDL933), they were absent in non-pathogenic E. coli (K-12 MG1655) (Additional file 3: Figure S2). These 17 HPIs corresponded to the membrane component of amino acid $\mathrm{ABC}$ transporter (YhdW). Earlier literatures have indicated at the potential of this membrane component of amino acid $\mathrm{ABC}$ transporter to act as a virulent factor. Evidences for the virulence potential of YhdW have previously been shown in different strains of Shigella and E. coli $[5,42,43]$.

\section{Salmonella-host interactions that are absent in the E. coli- host interactions}

The HPI profiles of the studied Salmonella strains (Additional file 1) shared as many as 418 HPIs with both pathogenic as well as non-pathogenic E. coli strains 


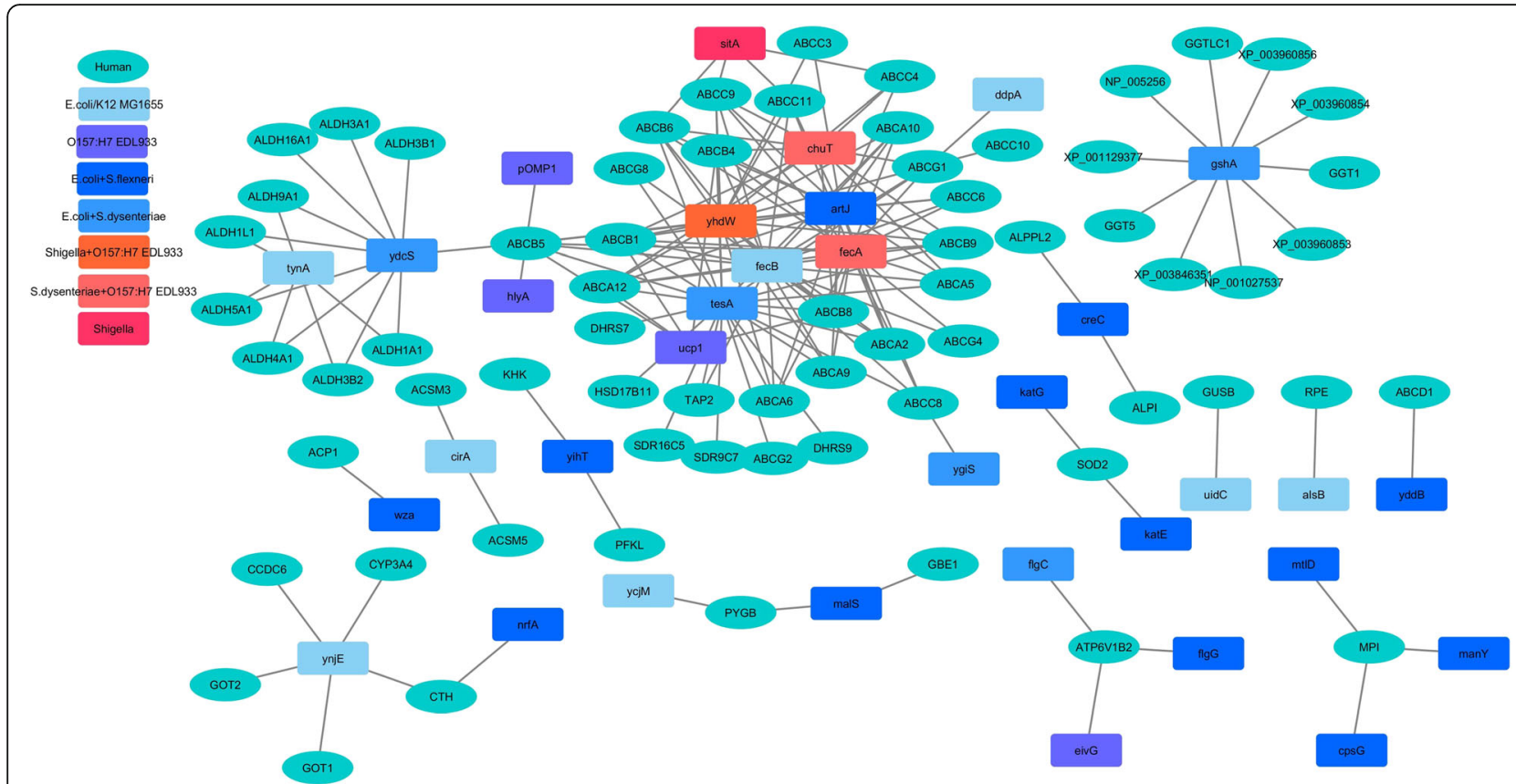

Fig. 3 Comparative analysis of the HPI-networks of studied Escherichia coli and Shigella strains. Depiction of the differences in the HPI network, along with the involved human (host) and bacterial (pathogen) proteins have been shown. 637 interactions that were common to all the studied stains of Escherichia coli and Shigella have not been shown in this figure

(Additional file 3: Figure S1). In addition, the three strains of Salmonella shared 146 interactions amongst themselves which were absent in the E. coli strains (Fig. 4 and Additional file 3: Figure S1). They include interactions pertaining to bacterial proteins like periplasmic murein peptide-binding protein (MppA), para-nitrobenzyl esterase (PnbA), and trifunctional nucleotide phosphoesterase protein $(\mathrm{YfkN})$. Periplasmic murein peptide-binding protein, MppA, which is known to functions as substratebinding protein, was seen to interact with human proteins involved in translocation of biliary lipids. Pertinently, enteric pathogens including Salmonella enterica are known to be resistant to the antibacterial properties of bile and are known to utilize bile salts as nutrients [44]. The paranitrobenzyl esterase, PnbA in Salmonella is known to catalyze the hydrolysis of several beta-lactam antibiotics. It was seen to interact with host carboxylesterases and proteins associated with heparan sulfate proteoglycan (HSPG). Given the multitude of roles played by HSPGs in immunity [45], it is likely that in addition to conferring antibiotic resistance, PnbA could also function in modulating the host immunopathology. Another Salmonella protein, namely trifunctional nucleotide phosphoesterase protein $\mathrm{YfkN}$, was found to interact with human proteins associated with nucleotide metabolism and seemed to be involved in the scavenging of nucleotides, particularly under conditions of phosphate shortage [46].

Among the human proteins which were involved in this subset of HPIs (with Salmonella), V-type proton
ATPase subunit B (ATP6V1B2) was found to be most interesting. Our observations indicate an intricate crosstalk between flagella biosynthesis pathway and Type III secretion system (T3SS) in Salmonella (Fig. 6). Support for this observation could be obtained from a previous literature which inspected the activation mechanisms of flagella biosynthesis and secretary systems in Salmonella [47]. Salmonella is known for its ability to arrest phagosomal maturation, thereby re-routing the maturation process towards formation of an invasion vacuole for its survival and replication [48]. Given the role of V-type proton ATPase in phagosomal maturation, it is likely that the re-routing of phagosomal maturation process in Salmonella infection is mediated through an ATP6V1B2 dependent process and is likely to include cross-talks between the pathogen's flagella biosynthesis and T3SS.

\section{Host-Vibrio interactions that are absent in Host-E. coli interactions}

Similar to the HPI profiles in Shigella and Salmonella strains, the studied strains of $V$. cholerae also exhibited 207 interactions which were not present in the HPI profiles of pathogenic as well as the non-pathogenic E. coli strains (Fig. 5 and Additional file 1). The possible biological significance of these interactions is discussed here. V. cholerae is known to use multiple strategies to acquire iron for its in vivo survival. This includes utilization of heme from hemoglobin as well as synthesis and transport of vibriobactin [49]. A membrane lipoprotein in V. cholerae, namely, 


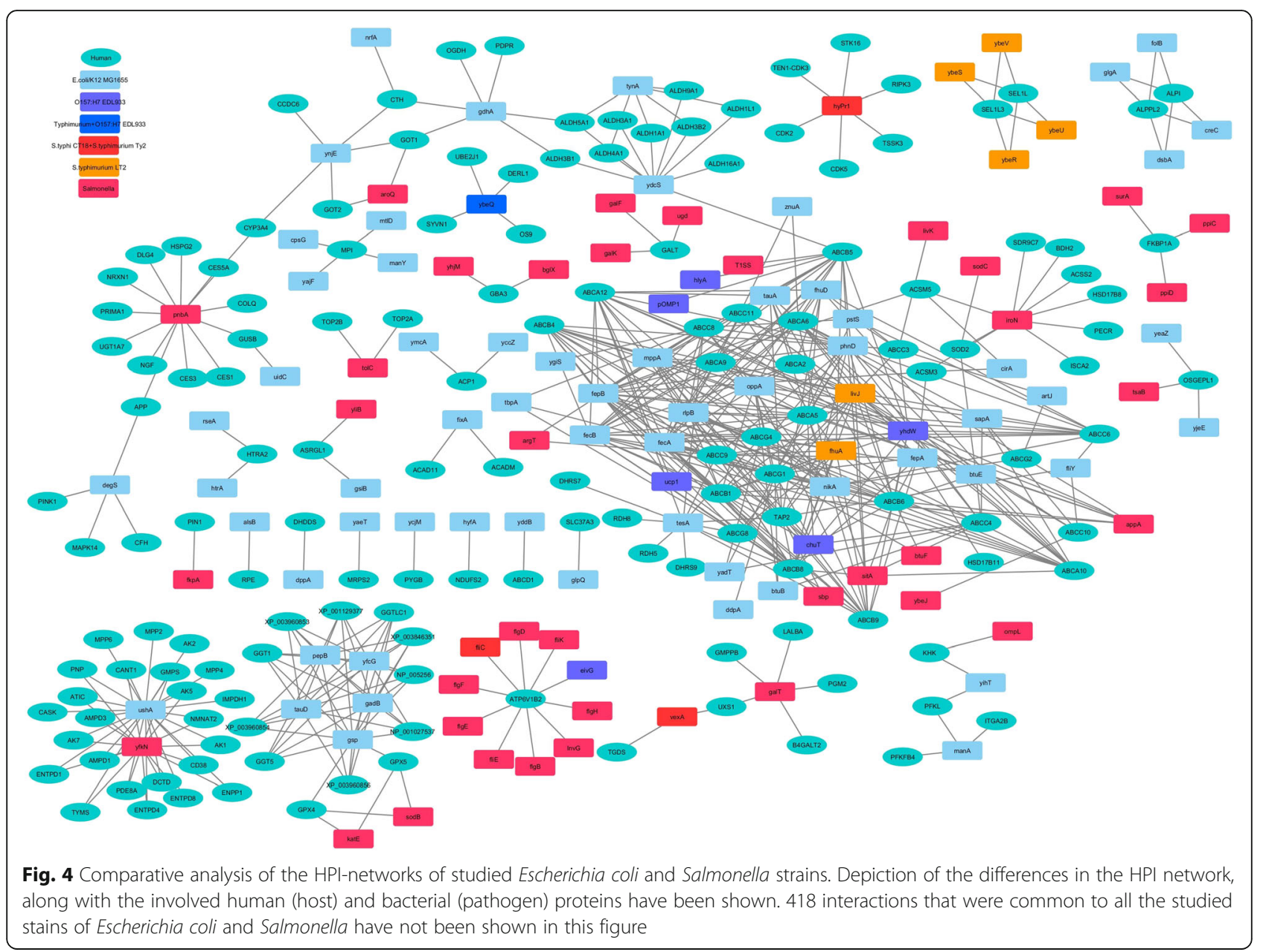

ferric vibriobactin-binding protein (ViuP), has previously been shown to function as a transporter for catechol siderophores (like vibriobactin), thereby aiding in iron acquisition [49]. In addition to the human $A B C$ transporters like phosphatidylcholine translocator (ABCB4), ATP-binding cassette sub-family $B$ member 6 (ABCB6), ATP-binding cassette sub-family $C$ member 9 (ABCC9), etc., ViuP was also seen to interact with human antigen peptide transporter 2 (TAP2) (Additional file 3: Figure S3). TAP2, in turn was seen to interact with a host of Vibrio proteins, including the $\mathrm{ABC}$ transporter ATP-binding proteins (AbcT1, AbcT2, AbcT3, and AbcT4), ferric vibriobactin enterobactin transport system substrate-binding protein (VctP), and periplasmic arginine-binding protein (ArtI) (Additional file 3: Figure S3). Notably, the human antigen peptide transporter 2 (TAP2) gene has been previously reported to be up-regulated during bacterial infections [50]. Previously published results coupled with the observations made in this work suggests TAP2 to be an important factor in the $V$. cholerae pathogenic process $[49,50]$.

\section{Host-pathogen interactions that are unique to only E. coli strains}

Overall, the interaction between proteins from host and those from the studied strains of Shigella, Salmonella and Vibrio were found to contain additional set of interactions which were not observed in the previously reported HPIs involving enteric E. coli strains [5]. However, the HPI data corresponding to the former set of organisms also seemed to lack several HPIs which were reported in case of both pathogenic as well as nonpathogenic E. coli strains [5]. It was therefore interesting to evaluate whether a subset of these unique interactions is crucial to the E. coli infection process. A subset of 18 HPIs was noted to be present only in the HPI profile of E. coli O157:H7 EDL933 (Fig. 1). Biological implication of some of these interactions has already been explained in an earlier literature [5]. In particular, HPIs involving bacterial proteins thiosulfate sulfurtransferase (YnjE) and T3SS outer membrane ring protein (EivG) were shown to be critical to the pathogenesis process [5]. 


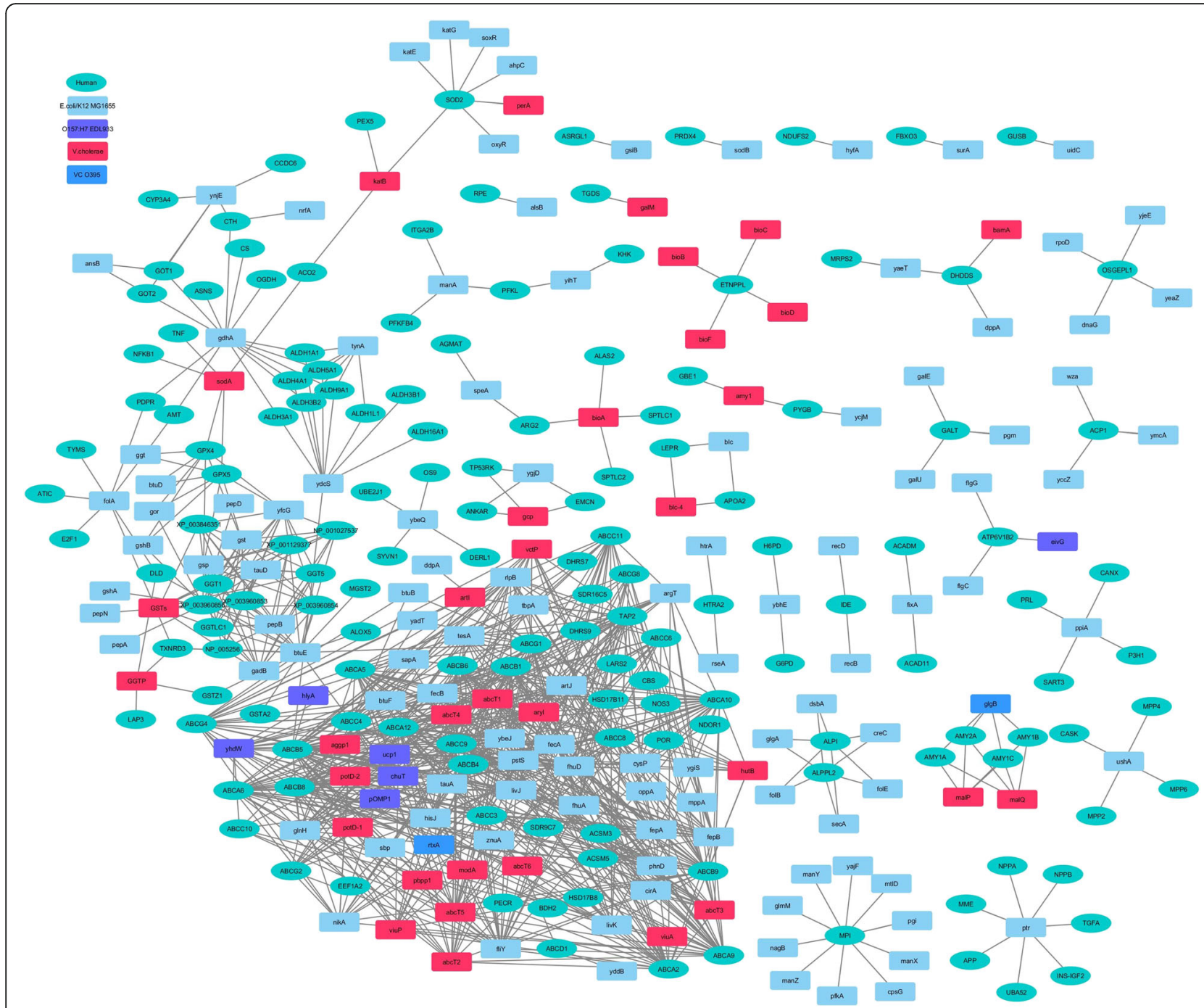

Fig. 5 Comparative analysis of the HPI-networks of studied Escherichia coli and Vibrio strains. Depiction of the differences in the HPI network, along with the involved human (host) and bacterial (pathogen) proteins have been shown. 139 interactions that were common to all the studied stains of Escherichia coli and Vibrio have not been shown in this figure

\section{Inter-species variations in interactions of enteric pathogens with host}

In addition to genus-specific variations in the HPI profiles of enteric pathogens, several inter-species differences in the HPI profiles were observed (Fig. 7 and Additional file 1). Some of those observed differences are discussed below.

\section{Inter-species variations in the HPI profiles of the Shigella strains}

While the HPI profiles of both the studied strains of Shigella shared majority of the interactions, a few speciesspecific interactions were also observed (Fig. 7a). A total of 77 HPIs were found to be uniquely present in S. dysenteriae when compared with S. flexneri 301 (Additional file 1). Among them, HPIs involving acyl-CoA thioesterase I
(TesA) were found to be particularly intriguing. Acyl-CoA Thioesterase I was seen to interact with human proteins 7dehydrocholesterol reductase (DHCR7) and hydroxysteroid 17-Beta Dehydrogenase 11 (HSD17B11) (Additional file 3: Figure S4). While 7-dehydrocholesterol reductase, DHCR7 is an enzyme involved in the conversion of 7 dehydrocholesterol to cholesterol, the hydroxysteroid 17Beta Dehydrogenase 11, HSD17B11 has been previously reported to be associated with the lipid droplets in the enterocytes [51].

In contrast to the above findings, 33 interactions were seen in the HPI profile of $S$. flexneri 301, which were absent in S. dysenteriae. Of them, the most notable were those involving $\mathrm{ABC}$ transporter arginine-binding protein 1 (ArtJ). ArtJ in S. flexneri 301 was found to be involved in 18 out of the 33 HPIs which were absent in $S$. 


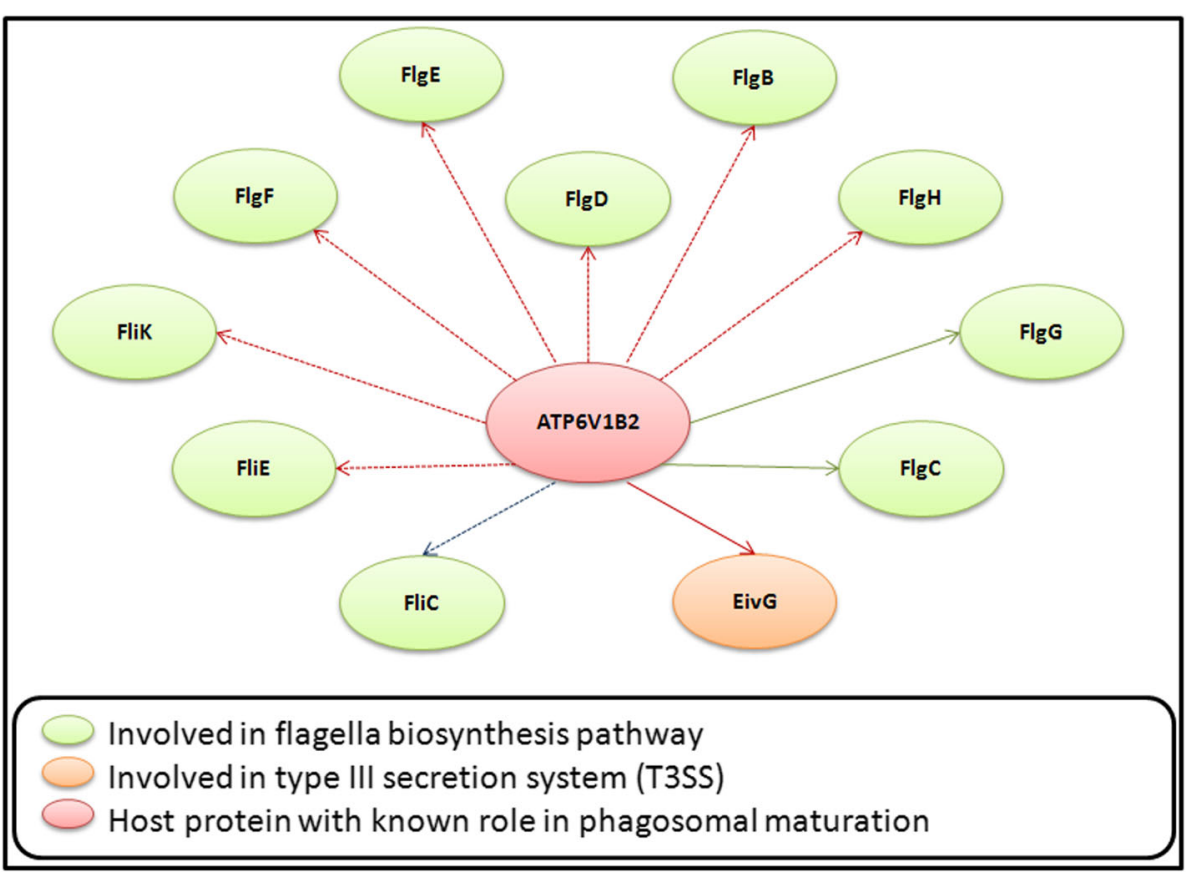

Fig. $6 \mathrm{HPIs}$ in Salmonella and E. coli involving human V-type proton ATPase subunit B (ATP6V1B2). ATP6V1B2 was involved in a total of 11 interactions with the Salmonella proteins. 10 of these interactions pertained to the flagella biosynthesis pathways in Salmonella. The HPI involving ATP6V1B2 and FliC (blue dashed arrow) was exclusive to S. enterica serovar Typhi strains (CT18 and Ty2). The remaining HPI (marked by red solid arrow) which involved EivG/ InvG (type III secretion apparatus protein) was observed in the HPI profile of all the studied Salmonella and E. coli strains except the non-pathogenic E. coli MG1655. Only two of the interactions involving flagella biosynthesis proteins (marked by solid green arrows) were seen in the HPI profiles of the pathogenic E. coli strains

dysenteriae (Additional file 1). Further, HPIs involving $\mathrm{ABC}$ transporter arginine-binding protein 1, ArtJ, were also seen to be present in all the other studied enteric pathogens (except $S$. dysenteriae), suggesting possible role of these interactions in pathogenesis by enteric bacteria. During the infection process, the most probable role ArtJ seems to be binding to arginine and sequestering of arginine from the host [52]. Notably, it was seen to interact with several host $\mathrm{ABC}$ transporter proteins. Pertinently, a recent publication which deliberated upon the role of enteric microbes in malnourishment in children indicated possibility of using arginine and glutamine supplements for improving health status [53]. In other words, it was indicated that malnourishment, at least in part, is linked to hijacking of essential nutrients (such as arginine and glutamine) by enteric pathogens, which otherwise feed into key host processes like nucleic acid biosynthesis and cellular replication [53]. The study further stated that malnourishment is also associated with disruption in the host's ability to transport/ uptake
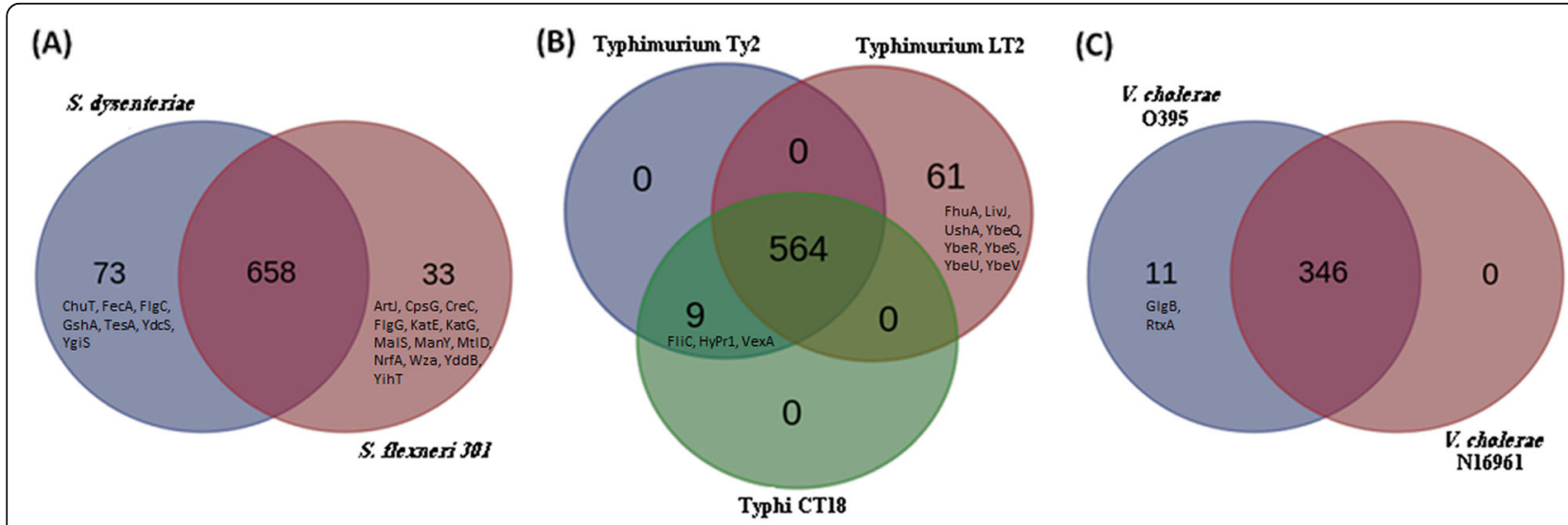

Fig. 7 Comparison of intra-species variations in the number of HPIs for the studied pathogens. a Shigella, b Salmonella, and c Vibrio 
nutrients. The observations made in the present work therefore echoes with reports from earlier literature and provide a possible mechanism through which essential nutrients like arginine are impounded by the enteric pathogens.

\section{Inter-species variations in the HPI profiles of the Salmonella strains}

Both the serovar Typhi strains (CT18 and Ty2) shared an identical set of proteins which interacted with the host proteins (Fig. 7b). In contrast, the S. enterica serovar Typhimurium LT2 demonstrated an additional repertoire of $61 \mathrm{HPIs}$, which were absent in the serovar Typhi strains. Majority of these interactions in S. enterica serovar Typhimurium LT2 were seen to involve one of the three bacterial proteins namely, ferrichrome-iron receptor (FhuA), Leu/Ile/Val-binding protein (LivJ), and UDP-sugar hydrolase (UshA). While FhuA and LivJ are possibly involved in nutrient uptake, UshA has been seen to be beneficial in evading host immune responses [31-33]. Furthermore, the rest of the interactions pertained to a set of poorly characterized bacterial proteins (namely, YbeQ, YbeR, YbeS/ DjlB, YbeU, and YbeV/ DjlC). Of these, YbeS (DjlB) and YbeV (DjlC) have been predicted to be associated to chaperone activity and YbeQ is a Sel1-repeat-containing protein. Notably, the subset of interactions involving YbeQ were also present in three of the five studied E. coli strains (EDL933, Sakai, MG1655) as well as the studied Shigella strains. It was even more interesting to observe that the aforementioned proteins were encoded in a single gene cassette in S. enterica serovar Typhimurium LT2. The only other (studied) organism which encode for the mentioned gene cassette was E. coli K-12 MG1655. The above observation when viewed in light of the fact that $S$. enteric serovar Typhimurium strains (which can infect different mammals) are less specific in infecting humans as compared to the serovar Typhi strains [54], it may be assumed that the set of HPIs involving the above mentioned poorly characterized bacterial chaperon and Sel1-repeat-containing proteins play little or no part in infections caused by enteric pathogens to humans. A deeper probe would however be required to establish this hypothesis.

\section{Inter-species variations in the HPI profiles of the Vibrio strains}

The HPI profiles of $V$. cholerae $\mathrm{O} 395$ contained 11 additional interactions as compared to that of $V$. cholerae $\mathrm{O} 1$ biovar El Tor N16961 (Fig. 7c). The subset of 11 HPIs involved two bacterial proteins, namely, multifunctionalautoprocessing repeats-in-toxin protein (RtxA), and 1,4alpha-glucan branching enzyme (GlgB). Given that multifunctional-autoprocessing repeats-in-toxin proteins are known to be present in both the studied strains of $V$. cholerae, the observed differences in HPI profiles were surprising. However, this apparent anomaly could have resulted due to the typical architecture of the RtxA protein. The toxin encoded by different strains of Vibrio are known to comprise of conserved and variable domains [55], and the observed variation in the HPI profiles were probably a reflection of the same. With respect to the HPIs involving 1,4-alpha-glucan branching enzyme, GlgB, it may be noted that glycogen is known to play an important role in the survival of $V$. cholerae, especially in nutrient poor aquatic environments [56]. GlgB is known to catalyze the gluconeogenesis process by creating branching of linear glucose chain (through cleavage of $1 \rightarrow 4$ bond and creation of $1 \rightarrow 6$ bond) [57]. It is therefore probable that the observed interactions between the host amylases and the bacterial 1,4-alpha-glucan branching enzymes are a result of the competition for similar substrates between the host and the invading pathogen. Given the fact that El Tor strains of $V$. cholerae (like N16961) are known to be more virulent than the classic strains (like O395) [58], the above observations may seem to be counter-intuitive. However, it may be noted that classical biotypes of $V$. cholerae have been observed to attain a viable but non-culturable (VBNC) state on co-culturing with El Tor biotypes [59]. It is therefore likely that in addition to the cross-talks with the host, closely related biotypes of $V$. cholerae may also interact among themselves. The outcome of the $V$. cholerae infection process is therefore not only dependent on its interaction with the host but is also influenced by the cross-talks among the infecting biotypes/ strains.

\section{Discussion}

Microbes which grow in similar ecological niche environments (e.g. inside human gut) are often seen to share a lot of common features that are essential to adapt to that environment [60]. Results presented through the in silico findings of this work also indicate a common repertoire of protein-protein interactions which were seen to be omnipresent in the host-pathogen interaction profiles of the studied pathogens belonging to genus Escherichia, Shigella, Salmonella and Vibrio. This subset of HPIs comprised of 122 interactions involving 122 host and 17 bacterial proteins. These 17 bacterial proteins may be considered as 'core factors' probably responsible for the bacteria to infect the host. However, these 122 interactions were also seen to be present in the HPI profile of the studied non-pathogenic E. coli strain. Hence, these set of HPIs should probably be regarded as 'niche' factors (rather than terming them as virulence factors), which provide an adaptive advantage to the inhabiting organisms inside the host. For example, ABC transporter periplasmic-binding protein, SapA, has previously been shown to be involved in the neutralization of 
antimicrobial peptides (AMPs), thereby aiding opportunistic pathogens like Haemophilus influenzae to infect the host [34]. Although previous literatures have linked the activity of SapA to pathogenesis, the current analysis indicates its participations even in the PPI networks involving non-pathogenic bacteria (E. coli MG1655). SapA therefore appears to be a more generic niche factor which is essential for the survival of most (enteric) bacterial species inside its host.

The HPI networks of the studied pathogens were seen to comprise of a few high degree nodes. Such nodes are expected to be involved in crucial biological functions. In other words, presence/ absence of such high degree nodes in the HPI network might translate to important variations in the infection processes. UDP-sugar hydrolase (UshA) and acyl-CoA thioesterase I (TesA) were identified as two such bacterial proteins which had high degree of interactions in the HPI network but was selectively absent in one or a few studied strains. UshA was found to be consistently present in the HPI profiles of studied bacterial strains, except in the two strains of S. enterica serovar Typhi. Based on previously published literature [31-33], we hypothesize that UshA may be involved in the hydrolysis of UDP-glucose. UDP-glucose and its receptor P2RY14 have previously been shown to be key players in triggering innate mucosal immune responses [61]. UshA may thus be associated with the capability to inhibit the innate immune system, thereby facilitating the bacterial infection process (Fig. 8). The absence of UshA in HPI-network (of S. enterica serovar Typhi strains) is indicative of possibly a different pathogenic mechanism in S. enterica serovar Typhi strains. It is probable that like in a few other gram negative intracellular pathogens [62], S. enterica serovar Typhi strains may be utilizing UDP-glucose to sequester energy in the nutrient limited environment within the vacuole.

Similarly, while acyl-CoA thioesterase I (TesA) was found to be absent in the HPI profile of Shigella flexneri 301, it was present in most other bacteria (including Shigella dysenteriae). TesA is a multifunctional enzyme that has thioesterase, lysophospholipase and protease activities [63-65] and inactivation/ absence of TesA has previously been reported to be associated with increased drug susceptibility and lipid metabolism [66]. TesA was seen to interact with human DHCR7 (7-dehydrocholesterol reductase), an enzyme involved in the conversion of 7-dehydrocholesterol to cholesterol (Additional file 3: Figure S4). Further, TesA was also seen to interact with human hydroxysteroid 17-Beta Dehydrogenase 11 (HSD17B11), which has been previously reported to be

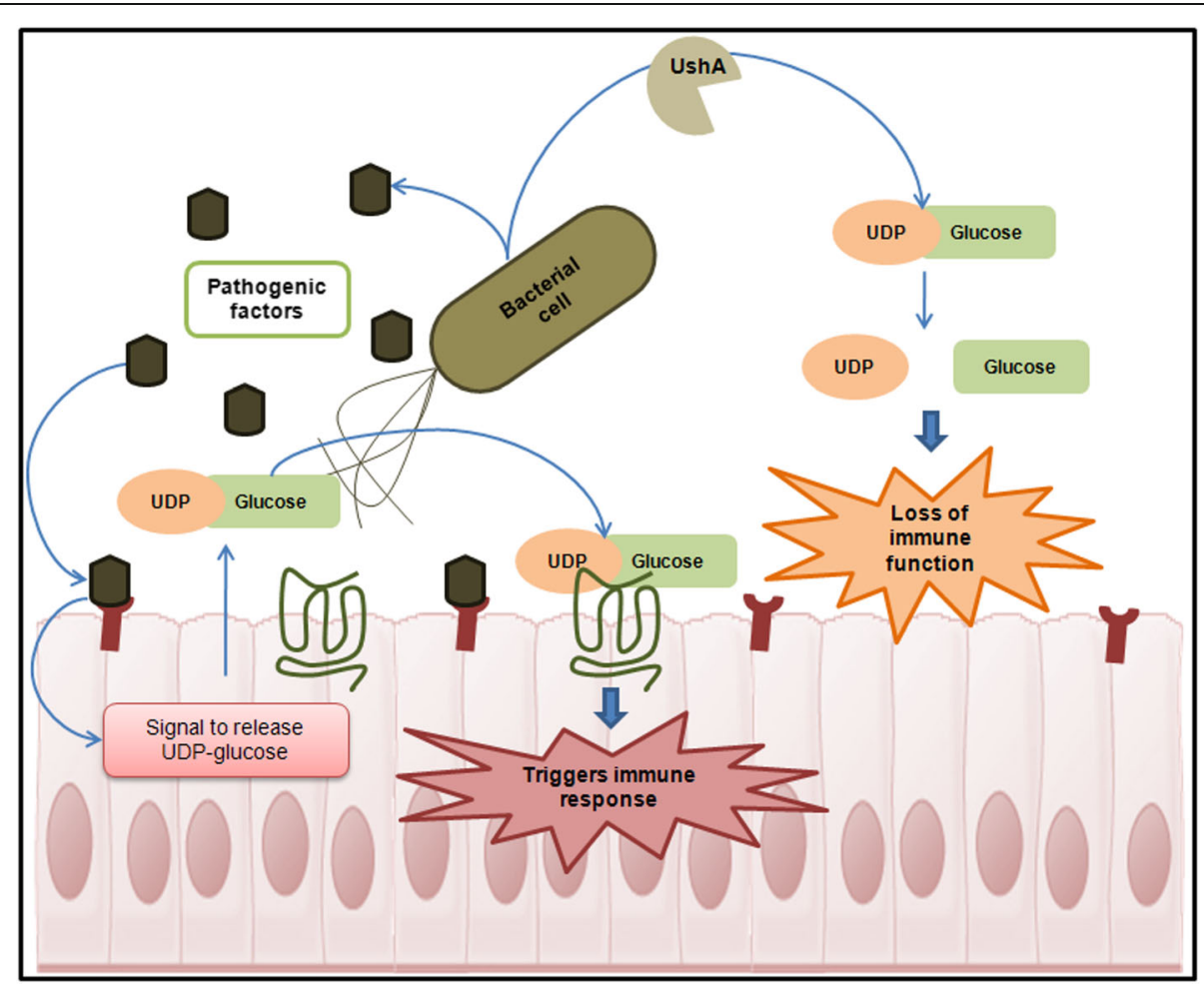

Fig. 8 Role of UDP-glucose in innate immune response and the probable UshA mediated mechanism of evading host defense by invading enteric pathogens. Human epithelial cells, in response to bacterial infection, release UDP-glucose. The released UDP-glucose acts as a signal to trigger innate immune responses. UshA, a UDP-glucose hydrolase can degrade the signaling molecule (UDP-glucose), and possibly acts as a virulence factor by abrogating the host defenses 
associated with the lipid droplets in the enterocytes [51]. While, the tesA gene in $S$. flexneri is known to be nonfunctional [67], it is pertinent to note that the initial stages of infection caused by most enteric pathogens (including Shigella) are often dependent on cholesterol and sphingolipids which are possibly acquired from the host enterocytes $[5,68]$. The above findings are possibly suggestive of the presence of an alternate functional machinery in S. flexneri that enables the pathogen to form lipid rafts to escape host defenses as well as attain resistance to antibacterial agents. However, since this is an in silico finding, additional experimental data would be required to validate this hypothesis.

In addition to the core set of HPIs, different groups of bacteria were seen to diverse strategies to best exploit the resources within the host micro environment. Interactions pertaining to a putative iron transport protein (SitA) in Shigella, a nucleotide phosphoesterase (YfkN), a para-nitrobenzyl esterase (PnbA) and a periplasmic peptide-binding protein (MppA) in Salmonella and ABC transporter ATP-binding proteins (AbcT1, AbcT2, AbcT3) in Vibrio are a few worth mentioning in this regard. While SitA in Shigella, MppA and YfkA in Salmonella and AbcTx in Vibrio were seen to be involved in acquisition of nutrients (like iron and phosphate) from the host, PnbA was seen to play roles in antibiotic resistance and immune modulation in Salmonella infection.

Given that these proteins do not have any homologues in humans, these bacterial proteins assume importance from the perspective of development of directed therapeutic strategies. Bacteria are more prone to developing (or acquiring) resistance to broad spectrum antibiotics, as compared to narrow spectrum antibiotics. In part, this could be attributed to horizontal transfer of genes within bacterial groups residing in a close association. Consequently, the strategy to target a set of proteins which are unique to small groups of bacteria and/ or are involved in interaction with the host in specific groups of bacteria is expected to reap benefits in the long run. The bacterial protein membrane component of amino acid ABC transporter (YhdW), which has previously been implicated in the pathogenicity of Shigella and E. coli $[5,42,43]$, and did not have any homologues in humans seemed to be a prospective therapeutic candidate against drug-resistant strains of Shigella and E. coli. Given that most of these proteins (which are involved in interaction with the host) are associated with the bacterial cell surface, the activity of these proteins can be restricted/ mitigated with relative ease using one or a combination of drug molecules.

It was also interesting to observe differences between the HPI profiles of (a) enteric pathogens as compared to a lung pathogen, and (b) gram positive and gram negative bacteria (see Additional file 3: Results section). In general, a higher number of proteins in gram negative bacteria were seen to be involved in interacting with the host proteins as compared to those in gram positive bacteria. For example, only 30 Mycobacterium tuberculosis H37Rv $(M t b)$ proteins were seen to interact with the host as compared to over a 100 (between 102 and 122) in most of the studied gram negative strains. Further, there was no significant overlap in set of bacterial proteins involved in HPI among the lung and the enteric pathogens. Only four bacterial proteins namely, gammaglutamyltransferase (Ggt), vitamin B12 import ATPbinding protein (BtuD), hydrogenase-4 component $\mathrm{A}$ (HyfA) and glucose-6-phosphate isomerase (Pgi) were found to occur in both the networks. When compared to the gram negative bacteria, the gram positive bacteria residing in the human gut were seen to share fewer interactions with the host. The above observations may partially be attributed to the higher number of ATPbinding cassette transporters ( $\mathrm{ABC}$ transporters) that are involved in interaction with the host in gram negative bacteria. The $\mathrm{ABC}$ transporters are involved in the active (energy driven) movement of molecules across the inner and outer membranes of the cell. Our observations concord with previous reports which suggested the occurrence of a higher number of surface proteins involved in macromolecule transport in gram negative bacteria as compared to their gram positive counterparts $[69,70]$. The outer membrane of gram negative bacteria which harbors several of the above mentioned proteins is also absent in gram positive bacteria $[69,70]$, thus explaining our observation of a higher number of HPI associated proteins in gram negative bacteria as compared to gram positive ones.

The HPIs reported in this work and the hypothesis derived from them were inferred on the basis of an in silico approach. Results obtained from such an in silico approach may contain a few false positive outcomes. Therefore, the confidence of the predicted HPIs (and their proposed mechanisms of actions) could have been improved if the method of predicting the HPIs was augmented with experimental evidences (gene/ protein/ metabolite expression data). The procedure had been adopted in one of the earlier works pertaining to the study of the survival of $M t b$ inside the human body [6]. However, the lack of suitable host and bacterial gene/ protein/ metabolite expression data prevented us from adopting the strategy in this case.

Further, the study identifies a few key bacterial proteins which may act as prospective therapeutic targets. While we have ascertained that these proteins do not share any homology with human proteins (data not shown), we could not test the essentiality of these bacterial gene products during in vivo survival, due to lack of adequate data. While popular knowledgebases like 
Database of Essential Genes (DEG) provide some information regarding gene essentiality, most of the data pertains to growth under rich nutrient medium which do not mimic the in vivo growth conditions prevalent inside a host.

In addition to HPIs, other virulent factors such as the bacteriotoxins may contribute to the pathogenesis of a disease-causing bacteria. Examples of such molecules include shiga toxins in E. coli and Shigella strains, choleragens in $V$. cholerae which can impact the degree of pathogenicity caused by the microbe. Cross-talks between the host and the pathogen involving such virulent factors were beyond the scope of this manuscript.

\section{Conclusion}

In spite of certain limitations, the current in silico study, possibly for the first time highlight a comparative analysis of HPI among different gut associated bacterial groups. Results presented herein provide insights into the bacterial processes that are possibly involved in the survival/ adaptation of various enteric pathogens inside the host body. The bacterial proteins which have been identified to be involved in interaction with host proteins (especially those which demonstrated high centrality measures in the HPI networks) could serve as attractive candidates for rational drug designing, thereby helping to tackle the menace of antibiotic resistance among bacterial pathogens.

\section{Methods}

The methodology adopted for identifying interacting hostpathogen protein pairs is similar to that used in our earlier published study [5]. The method is schematically depicted in Fig. 9 and details of the method have been provided in the Additional file 3: Materials and Methods section. In brief, human and bacterial protein sequences were first obtained from NCBI database (Additional file 3: Appendix 2). Using the BLASTClust program, a total of 16,599 unique clusters were identified among the bacterial protein sequences. Homologies between human and bacterial proteins were determined using BLASTp analysis. The most probable sub-cellular localization for the host and

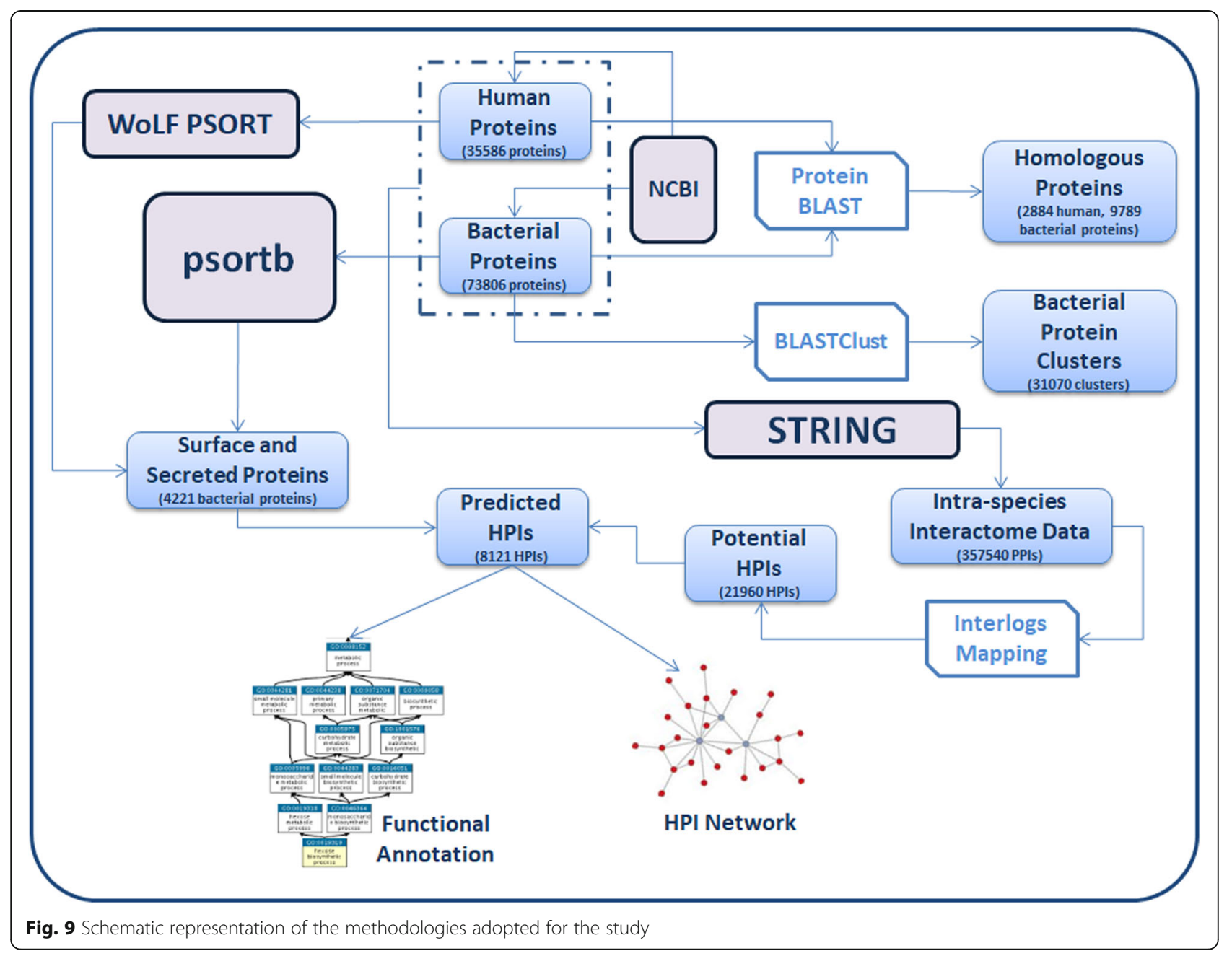


bacterial protein sequences were inferred using WoLF PSORT [71] and PSORTb version 3.0.2 [72] respectively. The HPIs were derived from information pertaining to (a) the template intra-species interaction data available from STRING database, version 9.1 [73] (http://string-db.org/) and (b) identified homology among human and bacterial proteins, (c) clustering of bacterial proteins, and (d) inferred sub-cellular localization of host and bacterial proteins. Subsequently, the host-pathogen interaction protein pairs were collated together to form HPI networks for each of the studied organisms and were analyzed for network properties using Cytoscape (version 2.8) [74] and CompNet [75]. The functional analysis of the proteins involved in HPIs was performed in terms of (i) Gene Ontology (GO) enrichment analysis, and (ii) KEGG functional pathway analysis. The GO enrichment analysis was performed using the data analysis module of STING web resource [76] (http://string-db.org/).

\section{Supplementary information}

Supplementary information accompanies this paper at https://doi.org/10. 1186/s12864-019-6398-2.

Additional file 1. List of predicted Host Pathogen Interactions (HPIs) for the studied host-pathogen models.

Additional file 2. Network statistics for each of the human and bacterial proteins which were associated to the host-pathogen interaction (HPI) network.

Additional file 3. Supplementary Methods, Results and Images.

Additional file 4. Gene Ontology analysis of the human and bacterial proteins associated to the host-pathogen interaction (HPI) network.

\footnotetext{
Abbreviations

ABCB4: $A B C$ transporters like phosphatidylcholine translocator $A B C B 4$; ABCB6: ATP-binding cassette sub-family B member 6; ABCC9: ATP-binding cassette sub-family C member 9; ACP1: Acid phosphatase 1;

AMPs: Antimicrobial peptides; Artl: Arginine-binding periplasmic protein 1; ATP6V1B2: V-type proton ATPase subunit B; BtuD: Vitamin B12 import ATPbinding protein; DEG: Database of Essential Genes; DHCR7: 7dehydrocholesterol reductase; EivG: Type III secretion system outer membrane ring protein; FhuA: Ferrichrome-iron receptor; Ggt: Gammaglutamyltransferase; GlgB: 1,4-alpha-glucan branching enzyme; Gor: Glutathione reductase; GshB: Glutathione synthetase; HPI: Host-Pathogen Protein-Protein Interaction; HSD17B11: Hydroxysteroid 17-Beta Dehydrogenase 11; HSPG: Heparan sulfate proteoglycan; HyfA: Hydrogenase-4 component A; LivJ: Leu/lle/Nal-binding protein; MppA: Periplasmic murein peptide-binding protein; MTB: Mycobacterium tuberculosis H37Rv; NLR: NODlike receptor; OSGEPL1: O-sialoglycoprotein endopeptidase like 1; PAMPs: Pathogen-associated molecular patterns; PepA: Cytosol aminopeptidase; PepD: Cytosol non-specific dipeptidase; PepN: Aminopeptidase N; Pgi: Glucose-6-phosphate isomerase; PnbA: Paranitrobenzyl esterase; PPI: Protein-Protein Interaction; ROS: Reactive oxygen species; RtxA: Multifunctional-autoprocessing repeats-in-toxin protein; SapA: ABC transporter periplasmic-binding protein; SitA: Putative iron transport protein SitA; SodB: Superoxide dismutase; T3SS: Type III secretion system; TAP2: Antigen peptide transporter 2; TbpA: Transferrin binding protein A; TesA: Acyl-CoA thioesterase l; UshA: UDP-sugar hydrolase; VBNC: Viable but non-culturable; ViuP: Ferric vibriobactin-binding protein; WHO: World Health Organization; YfkN: Trifunctional nucleotide phosphoesterase protein; YhdW: Amino acid ABC transporter; YnjE: Thiosulfate sulfurtransferase
}

\section{Acknowledgments}

We sincerely thank Dr. Anirban Dutta and Dr. Chandrani Bose for several helpful discussions and comments.

\section{Authors' contributions}

The work was conceptualized, and the experiments were designed by TB and SSM. TB performed the analysis. TB, SSM and KW analyzed the results and wrote the paper. All authors read and approved the final manuscript.

\section{Funding}

This work was partially supported by grants from the Department of Biotechnology (DBT), Government of India (BT/PR3260/BRB/10/ 967/2011). The funding body played no role in the design of the study and collection analysis, and interpretation of data and in writing the manuscript.

\section{Availability of data and materials}

The data sets generated and/or analysed in this study can be found in The National Center for Biotechnology Information database using the accession numbers listed in Additional file 3: Appendix 2.

\section{Ethics approval and consent to participate}

Not applicable.

\section{Consent for publication}

Not applicable.

\section{Competing interests}

Dr. Sharmila S. Mande and Mr. Tungadri Bose are employees of Tata Consultancy Services Limited. All other authors declare no competing interests.

\section{Author details}

${ }^{1}$ Bio-Sciences R\&D Division, TCS Innovation Labs, Tata Consultancy Services Limited, Pune, India. '2Department of Chemical Engineering, Indian Institute of Technology Bombay, Mumbai, India.

Received: 19 August 2019 Accepted: 15 December 2019

Published online: 27 December 2019

\section{References}

1. Guinane CM, Cotter PD. Role of the gut microbiota in health and chronic gastrointestinal disease: understanding a hidden metabolic organ. Ther Adv Gastroenterol. 2013;6:295-308.

2. Lozupone CA, Stombaugh Jl, Gordon Jl, Jansson JK, Knight R. Diversity, stability and resilience of the human gut microbiota. Nature. 2012;489: 220-30.

3. Jandhyala SM, Talukdar R, Subramanyam C, Vuyyuru H, Sasikala M, Reddy DN. Role of the normal gut microbiota. World J Gastroenterol. 2015;21: 8787-803.

4. WHO publishes list of bacteria for which new antibiotics are urgently needed. https://www.who.int/news-room/detail/27-02-2017-who-publisheslist-of-bacteria-for-which-new-antibiotics-are-urgently-needed. Accessed 7 Nov 2019.

5. Bose T, Venkatesh KV, Mande SS. Computational analysis of host-pathogen protein interactions between humans and different strains of Enterohemorrhagic Escherichia coli. Front Cell Infect Microbiol. 2017;7:128.

6. Bose T, Das C, Dutta A, Mahamkali V, Sadhu S, Mande SS. Understanding the role of interactions between host and mycobacterium tuberculosis under hypoxic condition: an in silico approach. BMC Genomics. 2018;19:555.

7. Rossez Y, Wolfson EB, Holmes A, Gally DL, Holden NJ. Bacterial flagella: twist and stick, or dodge across the kingdoms. PLoS Pathog. 2015;11:e1004483.

8. Mattoo S, Lee YM, Dixon JE. Interactions of bacterial effector proteins with host proteins. Curr Opin Immunol. 2007;19:392-401.

9. Ellermann M, Arthur JC. Siderophore-mediated iron acquisition and modulation of host-bacterial interactions. Free Radic Biol Med. 2017;105: 68-78.

10. Galán JE. Interaction of Salmonella with host cells through the centisome 63 type III secretion system. Curr Opin Microbiol. 1999;2:46-50.

11. Davis FP, Barkan DT, Eswar N, McKerrow JH, Sali A. Host pathogen protein interactions predicted by comparative modeling. Protein Sci Publ Protein Soc. 2007;16:2585-96. 
12. Huo T, Liu W, Guo Y, Yang C, Lin J, Rao Z. Prediction of host - pathogen protein interactions between mycobacterium tuberculosis and Homo sapiens using sequence motifs. BMC Bioinformatics. 2015;16:100.

13. Memišević V, Zavaljevski N, Rajagopala SV, Kwon K, Pieper R, DeShazer D, et al. Mining host-pathogen protein interactions to characterize Burkholderia mallei infectivity mechanisms. PLoS Comput Biol. 2015;11:e1004088.

14. Rapanoel HA, Mazandu GK, Mulder NJ. Predicting and analyzing interactions between mycobacterium tuberculosis and its human host. PLoS One. 2013;8:e67472.

15. Mahajan G, Mande SC. Using structural knowledge in the protein data bank to inform the search for potential host-microbe protein interactions in sequence space: application to mycobacterium tuberculosis. BMC Bioinformatics. 2017;18:201.

16. Zhou H, Rezaei J, Hugo W, Gao S, Jin J, Fan M, et al. Stringent DDI-based prediction of $\mathrm{H}$. sapiens-M. tuberculosis $\mathrm{H} 37 \mathrm{R} v$ protein-protein interactions. BMC Syst Biol. 2013;7(Suppl 6):S6.

17. Guven-Maiorov E, Tsai C-J, Ma B, Nussinov R. Interface-based structural prediction of novel host-pathogen interactions. Methods Mol Biol. 1851;2019:317-35 Clifton NJ.

18. Guven-Maiorov E, Tsai C-J, Ma B, Nussinov R. Prediction of host-pathogen interactions for helicobacter pylori by Interface mimicry and implications to gastric cancer. J Mol Biol. 2017:429:3925-41.

19. Guven-Maiorov E, Tsai C-J, Nussinov R. Structural host-microbiota interaction networks. PLoS Comput Biol. 2017;13:e1005579.

20. Dyer MD, Murali TM, Sobral BW. Computational prediction of host-pathogen protein-protein interactions. Bioinforma. 2007;23:i159-66 Oxf Engl.

21. Raman K, Bhat AG, Chandra N. A systems perspective of host-pathogen interactions: predicting disease outcome in tuberculosis. Mol BioSyst. 2010;6:516-30.

22. Cuesta-Astroz Y, Santos A, Oliveira G, Jensen LJ. Analysis of predicted hostparasite Interactomes reveals commonalities and specificities related to parasitic lifestyle and tissues tropism. Front Immunol. 2019;10. https://doi. org/10.3389/fimmu.2019.00212.

23. Devanga Ragupathi NK, Muthuirulandi Sethuvel DP, Inbanathan FY, Veeraraghavan B. Accurate differentiation of Escherichia coli and Shigella serogroups: challenges and strategies. New Microbes New Infect. 2017;21:58-62

24. Jeffery CJ, Koshland DE. Vibrio cholerae hlyB is a member of the chemotaxis receptor gene family. Protein Sci Publ Protein Soc. 1993;2:1532-5.

25. Kanehisa M, Sato Y, Kawashima M, Furumichi M, Tanabe M. KEGG as a reference resource for gene and protein annotation. Nucleic Acids Res. 2016:44:D457-62.

26. Kanehisa M, Goto S. KEGG: Kyoto encyclopedia of genes and genomes. Nucleic Acids Res. 2000;28:27-30.

27. Patil V, Kumar A, Kuruppath S, Nandi D. Peptidase N encoded by Salmonella enterica serovar Typhimurium modulates systemic infection in mice. FEMS Immunol Med Microbiol. 2007;51:431-42.

28. Ghezzi P. Role of glutathione in immunity and inflammation in the lung. Int J Gen Med. 2011;4:105-13.

29. Antoine Danchin antoine danchin [at] normalesup [dot]org. Sulfur metabolism in bacteria. 2011. http://www.normalesup.org/ adanchin/ science/sulfur-review.html. Accessed 1 Jan 2018.

30. Davidian J-C, Kopriva S. Regulation of sulfate uptake and assimilation - the same or not the same? Mol Plant. 2010;3:314-25.

31. AID_2013060511545426.pdf. http://file.scirp.org/pdf/AID_2013060511545426. pdf. Accessed 1 Jan 2018.

32. Lazarowski ER, Harden TK. UDP-sugars as extracellular signaling molecules: cellular and physiologic consequences of $\mathrm{P} 2 \mathrm{Y} 14$ receptor activation. Mol Pharmacol. 2015;88:151-60.

33. Sesma Jl, Weitzer CD, Livraghi-Butrico A, Dang H, Donaldson S, Alexis NE, et al. UDP-glucose promotes neutrophil recruitment in the lung. Purinergic Signal. 2016;12:627-35.

34. Shelton CL, Raffel FK, Beatty WL, Johnson SM, Mason KM. Sap transporter mediated import and subsequent degradation of antimicrobial peptides in Haemophilus. PLoS Pathog. 2011;7. https://doi.org/10.1371/journal.ppat.1002360.

35. Jo E-K, Kim JK, Shin D-M, Sasakawa C. Molecular mechanisms regulating NLRP3 inflammasome activation. Cell Mol Immunol. 2016;13:148-59.

36. Thinwa J, Segovia JA, Bose S, Dube PH. Integrin-mediated first signal for inflammasome activation in intestinal epithelial cells. J Immunol. 2014;193: 1373-82 Baltim Md 1950.
37. Martinon F. Detection of immune danger signals by NALP3. J Leukoc Biol. 2008;83:507-11.

38. Reis RSD, Horn F. Enteropathogenic Escherichia coli, Samonella, Shigella and Yersinia: cellular aspects of host-bacteria interactions in enteric diseases. Gut Pathog. 2010;2:8.

39. de Campos TA, Lago JC, Nakazato G, Stehling EG, Brocchi M, de Castro AF, et al. Occurrence of virulence-related sequences and phylogenetic analysis of commensal and pathogenic avian Escherichia coli strains (APEC). 2008.

40. Runyen-Janecky L, Reeves SA, Gonzales EG, Payne SM. Contribution of the Shigella flexneri sit, luc, and Feo iron acquisition systems to iron acquisition in vitro and in cultured cells. Infect Immun. 2003:71:1919-28.

41. Ulrich DL, Lynch J, Wang Y, Fukuda Y, Nachagari D, Du G, et al. ATPdependent mitochondrial porphyrin importer $A B C B 6$ protects against phenylhydrazine toxicity. J Biol Chem. 2012;287:12679-90.

42. Greenfield LK, Whitfield C. Synthesis of lipopolysaccharide O-antigens by ABC transporter-dependent pathways. Carbohydr Res. 2012;356:12-24.

43. Wallrodt I, Jelsbak L, Thorndahl L, Thomsen LE, Lemire S, Olsen JE. The putative thiosulfate sulfurtransferases PspE and GlpE contribute to virulence of Salmonella Typhimurium in the mouse model of systemic disease. PLoS One. 2013;8:e70829.

44. Urdaneta V, Casadesús J. Interactions between Bacteria and bile salts in the gastrointestinal and Hepatobiliary tracts. Front Med. 2017;4. https://doi.org/10.3389/fmed.2017.00163.

45. Simon Davis DA, Parish CR. Heparan sulfate: a ubiquitous glycosaminoglycan with multiple roles in immunity. Front Immunol. 2013;4. https://doi.org/10.3389/fimmu.2013.00470.

46. Chambert R, Pereira Y, Petit-Glatron MF. Purification and characterization of YfkN, a trifunctional nucleotide phosphoesterase secreted by Bacillus subtilis. J Biochem (Tokyo). 2003;134:655-60.

47. Das C, Dutta A, Rajasingh H, Mande SS. Understanding the sequential activation of type III and type VI secretion Systems in Salmonella typhimurium using Boolean modeling. Gut Pathog. 2013;5:28.

48. Brumell JH, Grinstein S. Salmonella redirects phagosomal maturation. Curr Opin Microbiol. 2004;7:78-84

49. Mey AR, Wyckoff EE, Oglesby AG, Rab E, Taylor RK, Payne SM. Identification of the Vibrio cholerae Enterobactin receptors VctA and IrgA: IrgA is not required for virulence. Infect Immun. 2002;70:3419-26.

50. Yang WE, Suchindran S, Nicholson BP, McClain MT, Burke T, Ginsburg GS, et al. Transcriptomic analysis of the host response and innate resilience to Enterotoxigenic Escherichia coli infection in humans. J Infect Dis. 2016;213:1495-504.

51. Beilstein F, Carrière V, Leturque A, Demignot S. Characteristics and functions of lipid droplets and associated proteins in enterocytes. Exp Cell Res. 2016:340:172-9.

52. Weerasinghe JP, Dong T, Schertzberg MR, Kirchhof MG, Sun Y, Schellhorn $\mathrm{HE}$. Stationary phase expression of the arginine biosynthetic operon $\operatorname{argCBH}$ in Escherichia coli. BMC Microbiol. 2006;6:14.

53. Guerrant RL, Oriá RB, Moore SR, Oriá MO, Lima AA. Malnutrition as an enteric infectious disease with long-term effects on child development. Nutr Rev. 2008;66:487-505.

54. Faucher SP, Curtiss R, Daigle F. Selective capture of Salmonella enterica Serovar Typhi genes expressed in macrophages that are absent from the Salmonella enterica Serovar Typhimurium genome. Infect Immun. 2005;73: 5217-21.

55. Dolores J, Satchell KJF. Analysis of Vibrio cholerae Genome Sequences Reveals Unique rtxA Variants in Environmental Strains and an rtxA-Null Mutation in Recent Altered El Tor Isolates. mBio. 2013;4:e00624-12.

56. Bourassa L, Camilli A. Glycogen contributes to the environmental persistence and transmission of Vibrio cholerae. Mol Microbiol. 2009:72:124-38.

57. Pal K, Kumar S, Sharma S, Garg SK, Alam MS, Xu HE, et al. Crystal structure of full-length mycobacterium tuberculosis H37Rv glycogen branching enzyme insights of N-terminal $\beta$-sandwich in substrate specificity and enzymatic activity. J Biol Chem. 2010;285:20897-903.

58. Son MS, Megli CJ, Kovacikova G, Qadri F, Taylor RK. Characterization of Vibrio cholerae $\mathrm{O} 1 \mathrm{El}$ Tor biotype variant clinical isolates from Bangladesh and Haiti, including a molecular genetic analysis of virulence genes. J Clin Microbiol. 2011:49:3739-49.

59. Pradhan S, Mallick SK, Chowdhury R. Vibrio cholerae classical biotype is converted to the viable non-Culturable state when cultured with the El Tor biotype. PLoS One. 2013;8:e53504. 
60. Hill C. Virulence or niche factors: What's in a name? J Bacteriol. 2012;194:5725-7.

61. Arase T, Uchida H, Kajitani T, Ono M, Tamaki K, Oda H, et al. The UDPglucose receptor P2RY14 triggers innate mucosal immunity in the female reproductive tract by inducing IL-8. J Immunol. 2009;182:7074-84 Baltim Md 1950.

62. Gehre L, Gorgette O, Perrinet S, Prevost M-C, Ducatez M, Giebel AM, et al. Sequestration of host metabolism by an intracellular pathogen. eLife. 2016;5:e12552

63. Doi O, Nojima S. Lysophospholipase of Escherichia coli. J Biol Chem. 1975;250:5208-14.

64. Cho H, Cronan JE. "Protease I" of Escherichia coli functions as a thioesterase in vivo. J Bacteriol. 1994;176:1793-5.

65. Lee YL, Chen JC, Shaw JF. The thioesterase I of Escherichia coli has arylesterase activity and shows stereospecificity for protease substrates. Biochem Biophys Res Commun. 1997;231:452-6.

66. Chavadi SS, Edupuganti UR, Vergnolle O, Fatima I, Singh SM, Soll CE, et al. Inactivation of tesA reduces Cell Wall lipid production and increases drug susceptibility in mycobacteria. J Biol Chem. 2011;286:24616-25.

67. Jin $Q$, Yuan Z, Xu J, Wang Y, Shen Y, Lu W, et al. Genome sequence of Shigella flexneri 2a: insights into pathogenicity through comparison with genomes of Escherichia coli K12 and O157. Nucleic Acids Res. 2002;30:4432-41.

68. Lafont F, Tran Van Nhieu G, Hanada K, Sansonetti P, van der Goot FG. Initial steps of Shigella infection depend on the cholesterol/sphingolipid raftmediated CD44-IpaB interaction. EMBO J. 2002;21:4449-57.

69. Yen MR, Peabody CR, Partovi SM, Zhai Y, Tseng YH, Saier MH. Proteintranslocating outer membrane porins of gram-negative bacteria. Biochim Biophys Acta. 2002;1562:6-31.

70. Lubelski J, Konings WN, Driessen AJM. Distribution and physiology of ABCtype transporters contributing to multidrug resistance in Bacteria. Microbiol Mol Biol Rev. 2007;71:463-76.

71. Horton P, Park K-J, Obayashi T, Fujita N, Harada H, Adams-Collier CJ, et al. WoLF PSORT: protein localization predictor. Nucleic Acids Res. 2007;35(Web Server issue):W585-7.

72. Yu NY, Wagner JR, Laird MR, Melli G, Rey S, Lo R, et al. PSORTb 3.0: improved protein subcellular localization prediction with refined localization subcategories and predictive capabilities for all prokaryotes. Bioinformatics. 2010;26:1608-15.

73. Franceschini A, Szklarczyk D, Frankild S, Kuhn M, Simonovic M, Roth A, et al. STRING v9.1: protein-protein interaction networks, with increased coverage and integration. Nucleic Acids Res. 2013;41:D808-15.

74. Shannon P, Markiel A, Ozier O, Baliga NS, Wang JT, Ramage D, et al. Cytoscape: a software environment for integrated models of biomolecular interaction networks. Genome Res. 2003;13:2498-504.

75. Kuntal BK, Dutta A, Mande SS. CompNet: a GUI based tool for comparison of multiple biological interaction networks. BMC Bioinformatics. 2016;17:185.

76. Szklarczyk D, Franceschini A, Wyder S, Forslund K, Heller D, Huerta-Cepas J, et al. STRING v10: protein-protein interaction networks, integrated over the tree of life. Nucleic Acids Res. 2015;43:D447-52.

\section{Publisher's Note}

Springer Nature remains neutral with regard to jurisdictional claims in published maps and institutional affiliations.

Ready to submit your research? Choose BMC and benefit from:

- fast, convenient online submission

- thorough peer review by experienced researchers in your field

- rapid publication on acceptance

- support for research data, including large and complex data types

- gold Open Access which fosters wider collaboration and increased citations

- maximum visibility for your research: over $100 \mathrm{M}$ website views per year

At BMC, research is always in progress.

Learn more biomedcentral.com/submissions 